\title{
Erratum
}

\section{Ann. Surg. Oncol., Vol. 13, No. 2, February 2006, pp. 140-141 (DOI: 10.1245/ASO.2006.07.028)}

In the article by Kemeny, entitled "Expressing the Surgery First Position on Treatment of Synchronous Colorectal Metastases in the Asymptomatic Patient," the title appeared incorrectly.

The correct title of the article is:

"Surgery Should Be the Primary Treatment of Synchronous Colorectal Metastases in the Asymptomatic Patient"

Published online: March 2, 2006. 